\title{
Obstructive Sleep Apnea - Insomnia Overlap Syndrome
}

\author{
${ }^{1}$ Unnati Desai, ${ }^{2}$ Jyotsna M Joshi
}

\begin{abstract}
Sleep-disordered breathing and insomnia are the most common of the sleep disorders. Both these diseases are well defined, diagnosed and managed as per the current guidelines. The initial isolated reports of an overlap of these two diseases were published in the eighties. With newer studies post-2001, the existence of the comorbid obstructive sleep apnea (OSA) and upper airway resistance syndrome (UARS) with insomnia has been increasingly recognized. This disease entity has been referred with diverse unstandardized terminologies. UARS is a variant nomenclature of OSA as per International Classification of Sleep Disorders- third edition (ICSD-3). We hereby address it as the OSA-insomnia overlap syndrome throughout the text and review literature. Alike the ununiform terminology, there is lack of clear textbook/guideline definitions, diagnosis, and management of the OSA-insomnia overlap syndrome. Both have an interlinked pathophysiology. The clinical features and evaluation of the OSA-insomnia overlap syndrome share similarities to the diseases in isolation with exacerbation of overlapping features. An active search for the diagnosis of the overlap syndrome in patients visiting the sleep apnea clinic and insomnia clinic is mandatory as the identification helps in optimizing the management and difficulties in poor responders/ failures. Continuous positive airway pressure (CPAP) therapy with cognitive/behavior therapy for insomnia (CBTi) is the treatment of choice. A lot is yet to surface in the research of the OSA-insomnia overlap syndrome.
\end{abstract}

Keywords: CBT, CPAP, Insomnia, OSA, Overlap, SDB

How to cite this article: Desai U, Joshi JM. Obstructive Sleep Apnea-Insomnia Overlap Syndrome. Indian Sleep Med 2018;13(1):13-17.

Source of support: Nil

Conflict of interest: None

\section{INTRODUCTION}

Sleep-disordered breathing (SDB) encompasses a wide

\footnotetext{
${ }^{1}$ Associate Professor, ${ }^{2}$ Professor and Head

${ }^{1}$ Department of Pulmonary Medicine, Topiwala National Medical College \& BYL Nair Charitable Hospital, Mumbai, India

${ }^{2}$ Department of Pulmonary Medicine, Topiwala National Medical College \& BYL Nair Charitable Hospital, Mumbai, India

Corresponding Authors: Jyotsna M Joshi, Professor and Head, Department of Pulmonary Medicine, Topiwala National Medical College \& BYL Nair Charitable Hospital, Mumbai, India, Tel: 02223027643, e-mail: drjoshijm@gmail.com
}

range of sleep-related respiratory abnormalities, characterized by repeated pauses in breathing during sleep or abnormalities in ventilation during sleep. ${ }^{1-3}$ When SDB is combined with characteristic sleep-related symptoms such as excessive daytime sleepiness, daytime fatigue, and unrefreshing sleep, it defines the obstructive sleep apnea syndrome (OSAS) ${ }^{1-3}$ Insomnia is the most common sleep disorder with variable prevalence rates reported. Insomnia literally means lack of sleep at bedtime. Insomnia classifications and definitions have undergone multiple revisions. Insomnia is considered to be a state of hyperarousal which may be a primary or secondary disorder. While acute insomnia is limited and reversible. Chronic insomnia is characterized by a subjective complaint of insomnia (regarding the amount and/or quality of sleep) and daytime problems of a physiological, emotional and cognitive nature. ${ }^{1-3}$ Approximately half of the patients with SDB also experience insomnia. ${ }^{1-3} \mathrm{On}$ the other side, insomnia has been reported to have associated with comorbid obstructive sleep apnea (OSA). ${ }^{1-4}$ The cause and effect of both have been debated. The association between SDB and chronic insomnia has long been recognized. In 1973, Guilleminault and colleagues first described a sleep apnea-insomnia syndrome in two male patients who complained of chronic multiple arousals and insomnia for many years. ${ }^{5}$ Three years later, the same group reported in a case series analysis that 6 out of 56 patients $(10.7 \%)$ with nighttime snoring and chronic insomnia had the sleep apnea-insomnia syndrome and $61 \%$ of patients had central sleep apnea. ${ }^{6}$ More recently, Krakow et al. re-highlighted this problem. ${ }^{7}$ Sufficient evidence has now accumulated to warrant conceptualization of comorbid insomnia and SDB as a distinct clinical syndrome. Also called complex SDB or Insomnia-Sleep Disordered Breathing Syndrome. ${ }^{1-3}$ Insomnia and SDB appear to uniquely contribute to the morbidity of these patients. Associated SDB described included mainly OSA and upper airway resistance syndrome (UARS). The ICSD-3 emphasized that UARS is a variant of OSA. Hence, for further reference in the text, we use the terminology of OSA-insomnia overlap syndrome. We review the literature till date about this emerging disease.

\section{DEFINITION AND DIAGNOSTIC CRITERIA}

Insomnia has numerous definitions and is characterized by disturbed or decreased sleep in the presence of 
opportunity and circumstances for sleep..$^{8,9}$ The ICSD- $3^{8}$ diagnostic criteria for chronic insomnia disorder concur with the general criteria for an insomnia disorder in ICSD-2. ${ }^{9}$ They include (1) sleep initiation or maintenance problems, (2) adequate opportunity and circumstances to sleep, and (3) daytime consequences at least thrice a week for a duration of three months. There are various validated indices that measure the insomnia severity like the Athens insomnia scale ${ }^{10}$ and insomnia severity index. ${ }^{11}$ The first description of OSA was given by the novelist Charles Dickens in 1836. The terminology of OSAS was coined by Guilleminault in 1976. Sleep-related breathing disorders are divided into four: OSAS, central sleep apnea (CSA) syndromes, sleep-related hypoventilation disorders, and sleep related hypoxemia disorder. ${ }^{12}$ The core criteria for a diagnosis of OSA defined in ICSD-2 are valid in the ICSD-3. The diagnosis requires either signs/symptoms (e.g., sleepiness, fatigue, insomnia, snoring, subjective nocturnal respiratory disturbance, or observed apnea) or associated medical or psychiatric disorder (i.e., hypertension, coronary artery disease, atrial fibrillation, congestive heart failure, stroke, diabetes, cognitive dysfunction, or mood disorder) coupled with five or more predominantly obstructive respiratory events (obstructive and mixed apneas, hypopneas, or respiratory effort-related arousals, as defined by the AASM scoring manual) per hour of sleep during polysomnography (PSG). Alternatively, a frequency of obstructive respiratory events more than and equal to fifteen per hour satisfies the criteria, even in the absence of associated symptoms or disorders. The ICSD-3 allows the use of an out-of-center sleep testing (OCST) for the diagnosis. ${ }^{8,9,12}$ There is no consensus on the definition and diagnostic criteria of the OSA-insomnia overlap syndrome.

\section{INITIAL STUDIES}

OSA and insomnia are the most common sleep disorders. While OSA is characterized by excessive sleepiness; insomnia has sleeplessness. The antonymic symptoms with the contrasting differences in conceptualizations of both these diseases have led to under-appreciation of the overlap in spite of its documentation in the early eighties. ${ }^{13}$ Studies have reported a $30-70 \%$ of OSA patients to have insomnia. ${ }^{14}$ The concept is now better studied and multiple randomized control trials are underway. After the initial description by Guilleminault et al. in 1973 and 1976, Krakow and colleagues reported the comorbidity in 2001 in a retrospective study. Amongst their 231 patients with SDB diagnosed by PSG, the SDB-plus (insomnia) patients had statistically significant complaints of difficulty falling asleep or staying asleep, longer mean sleep latency, shorter total sleep time, impaired sleep efficiency, restless legs or leg jerks, poor sleep quality as compared to SDB-only patients. ${ }^{7}$ A 2004 study by Smith et al. showed strong correlation of comorbid insomnia symptom severity with OSA severity. ${ }^{15}$ Krakow et al. reported a pilot study in 2004 for further research in the comorbidity. ${ }^{16}$ In contrast, in 2005, Krell et al. reported vice versa. In their study, insomnia complaints were more common in patients without significant SDB than in those with SDB. The most common insomnia complaints reported were sleep-maintenance insomnia, sleep onset insomnia and insomnia with early awakening. ${ }^{17}$ Chung determined the prevalence of various insomnia subtypes in 157 patients with diagnosed OSA and found that $91(58.0 \%)$ had no sleep problems, while $44(28.0 \%)$ had one insomnia symptom, 15 (9.6\%) had two insomnia symptoms, and $7(4.4 \%)$ had three or four insomnia symptoms with sleep maintenance insomnia as the most common complaint. ${ }^{18}$ These studies further promoted and laid the stepping stones for the systematic research of OSA-insomnia overlap syndrome the results of which are eagerly awaited.

\section{PATHOPHYSIOLOGY}

The developmental course of the OSA-insomnia overlap is not yet clearly understood and several theories are hypothesized. Both disorders are thought to be an effect and cause of the overlap. Traditionally, insomnia symptoms were hypothesized to respiratory disturbance in OSA. Hence, the focus of therapy in yesteryears was only OSA. However, OSA has now been identified through multifactorial reasons to predispose for insomnia. OSA is characterized by post-apneic arousals which cause increased night awakening leading to sleep dissatisfaction and cause insomnia. OSA is associated with nocturia causing repeated awakening, poor sleep hygiene and lead to insomnia. OSA patients may overuse caffeine, increase the time in bed, habituate to daytime napping to decrease sleepiness. All these habits are established theories to cause insomnia. OSA patients may perceive repeated short arousals as wakefulness. This may activate the sympathetic nervous system and cause anxiety which may develop psychosocial/learned insomnia. OSA therapy with continuous positive airway pressure (CPAP) may unmask underlying insomnia. Insomnia exacerbates OSA through various mechanisms. Insomnia causes abrupt wake-sleep transitions, sleep deprivation, sleep fragmentation that may cause OSA. Insomnia associated prolonged awakening causes sudden abrupt airway collapse leading to OSA. The stress-induced activation of the hypothalamic-pituitary (HPA) axis has been hypothesized to be a major pathway through which symptoms of OSA and insomnia cause and increase 
the risk of each other. ${ }^{1,2,3,13,14}$ When OSA and insomnia overlap, each condition has an independent contribution to overall morbidity, and targeted therapy of each condition is necessary to achieve satisfactory outcomes in these cases. ${ }^{3}$ Figure 1 depicts the pathophysiology of the OSA-insomnia overlap syndrome.

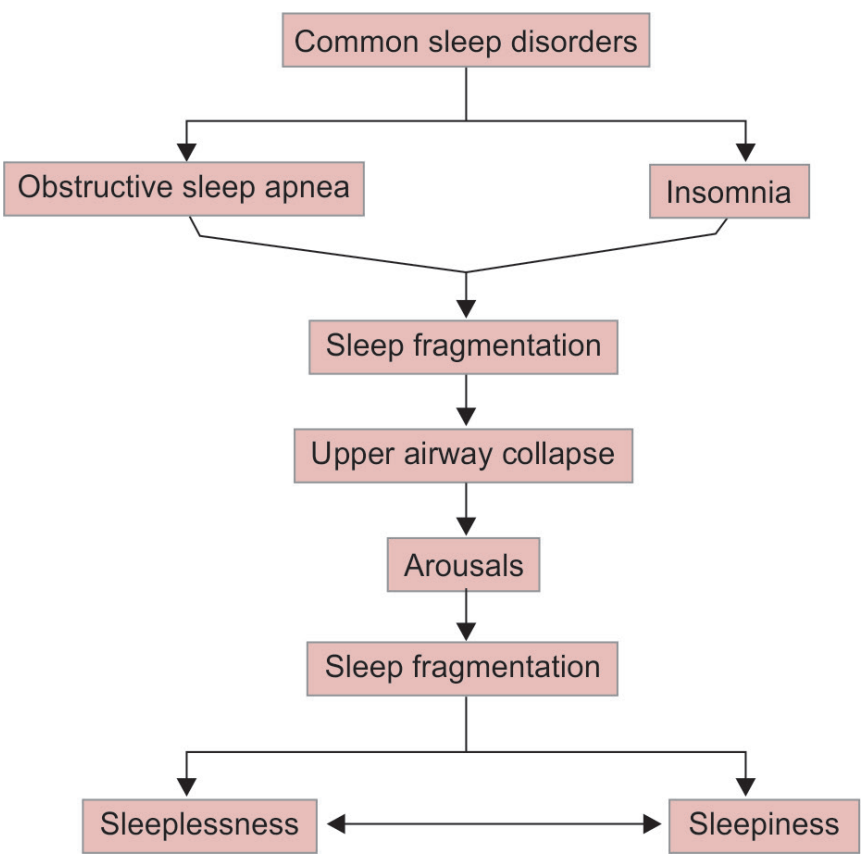

Fig. 1: Pathophysiology of OSA-insomnia overlap syndrome

\section{CLINICAL FEATURES}

The symptomatology of both disorders has distinct as well as overlapping features. Certain clinical symptomatology and questionnaire's validity and specificity for individual disorders of OSA and insomnia have been challenged as more is unearthed about the OSA-insomnia overlap syndrome. OSA is characterized by snoring, witnessed apnea, choking episodes, gasping, sleep arousals, dry mouth sensation, morning headache and excessive daytime somnolence (EDS). The Epworth sleepiness scale is an effective tool to measure the EDS. Also, various pre-test probability scores for OSA like the sleep apnea clinical score (SACS), BERLIN, STOP-BANG and the adjusted neck circumference score (ANCS) quantify the pre-test probability of OSA. The pre-test probability scores may be a useful tool in resource-limited settings to identify patients with priority for PSG. Insomnia specific symptoms include sleep initiation problems, sleep anxiety, dysfunctional attitudes and beliefs about sleep, sleep anxiety, learned sleep prevention, and sleeplessness. The insomnia severity index and sleep wake diary are effective tools to grade insomnia. The symptoms which overlap both diseases and are more significant in the OSA-insomnia overlap group of patients include fatigue, non-refreshing sleep, impaired attention and concentration, mood dysfunction, sleep maintenance problems, impaired quality of life, absenteeism from work, increased associations with depression, anxiety and other psychiatric disorders with increased utilization of healthcare resources. ${ }^{1-5}$ Figure 2 depicts the symptomatology of OSA, insomnia and OSA-insomnia overlap syndrome. Future research is to measure and validate existing tools of diagnosis, develop new questionnaire to identify the OSA and insomnia disorders in isolation and concurrently to guide management.

\section{EVALUATION AND DIAGNOSIS}

All patients visiting the sleep apnea/pulmonologist and insomnia/psychiatrist clinics should be screened for the comorbid conditions either at baseline or followup as per the logistics of the treating facility. One of the comorbid conditions may predominate at presentation and is usually the focus of evaluation and treatment. While baseline clinical assessment of the primary problem (OSA/insomnia) is already available, further

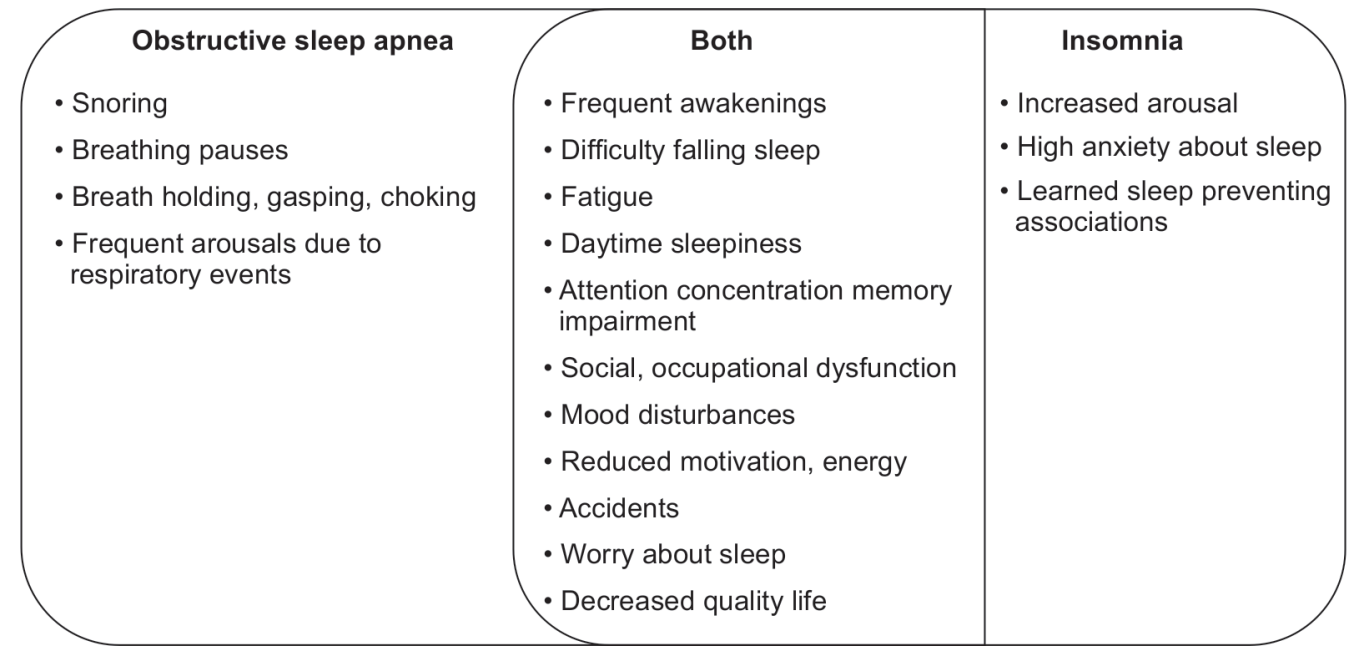

Fig. 2: Symptomatology of OSA-insomnia overlap syndrome 
screening assessment for the comorbidity should be actively pursued wherever cross-referral services are available in the tertiary care centers. In secondary referral centers or clinics where cross-referrals are difficult, the patient should be initiated on therapy of the primary condition and reassessed at follow-up. OSA patients should be screened for insomnia with screening questions of sleep initiation, maintenance, and awakening. The predominance of excessive daytime sleepiness over fatigue or vice versa should be assessed. The severity of insomnia can be assessed by the insomnia severity index. Vice-versa insomnia patients should be screened with a clinical history, examination and pre-test probability sleep scores followed by a PSG. An ideal treatment algorithm is illustrated in Figure 3.,13,14

\section{MULTIDISCIPLINARY MANAGEMENT}

The management of OSA-insomnia overlap syndrome requires optimal treatment of both the disorders. Whether, when and which therapy should be prioritized or both therapies should be initiated simultaneously is the topic of research for the forthcoming trials. The management first and foremost requires a correct identification of the overlap syndrome. To identify correctly, the concerned specialty clinics should be appraised of the data and prevalence of this disorder and encourage suspicion in every relevant case scenario. This requires derivation of validated, standardized, easy to use screening questionnaires to be used in the sleep apnea and insomnia clinics to identify the OSA-insomnia overlap syndrome. The current questionnaires for OSA and insomnia have been devised when the information on the overlap was inexistent. Hence, they are unreliable and irrelevant to identify / screen for an overlapping disorder. If manifestations of one of the disease entities predominate clinically, then logically management of the same should be initiated first. Reassessment of improvement of the predominant condition and existing symptoms of the second condition should be done timely at follow-up. Associated other comorbid medical conditions should be optimized. OSA is managed with respect to severity of symptoms in addition to the supportive management. Mild OSA can be managed with weight loss, avoidance of sleep deprivation, sedatives, narcotics, alcohol, positional therapy. Moderate and severe OSA is managed with CPAP therapy, etc. and lifestyle modifications. ${ }^{12}$ Oral appliances have a limited role in mild OSA. Surgery (uvulopalatopharyngoplasty) though useful is usually the last resort if CPAP cannot be used. Adenotonsillar hypertrophy resultant OSA, especially in children is managed effectively with adenotonsillectomy.

Insomnia therapy involves pharmacotherapy and nonpharmacotherapy. Earlier studies have recommended against the use of hypnotics in comorbid OSA patients due to their muscle relaxant action and alpha-hypopnea index (AHI) exacerbation. As regards the OSA-insomnia overlap syndrome, whether the use of newer drugs which do not affect $\mathrm{AHI}$ is beneficial or not is still a matter of research. Pharmacotherapy for insomnia provides symptomatic relief but has disadvantages of rebound insomnia, daytime sedation, slowed reactions, memory impairment, and amnesia. Hence, nonpharmacotherapy is highlighted as the treatment of choice. It is also to be used in the OSA-insomnia overlap syndrome. Cognitive/behavior therapy for insomnia (CBTi) is the generic terminology used for a combination of different therapeutic elements that consist of the nonpharmacotherapy. CBTi has been shown to be effective in randomized controlled clinical trials as compared to pharmacotherapy. ${ }^{19}$ The behavioral therapies aimed at changing behavior and establishing new associations to treat insomnia. The most applied behavior change therapies are stimulus control therapy

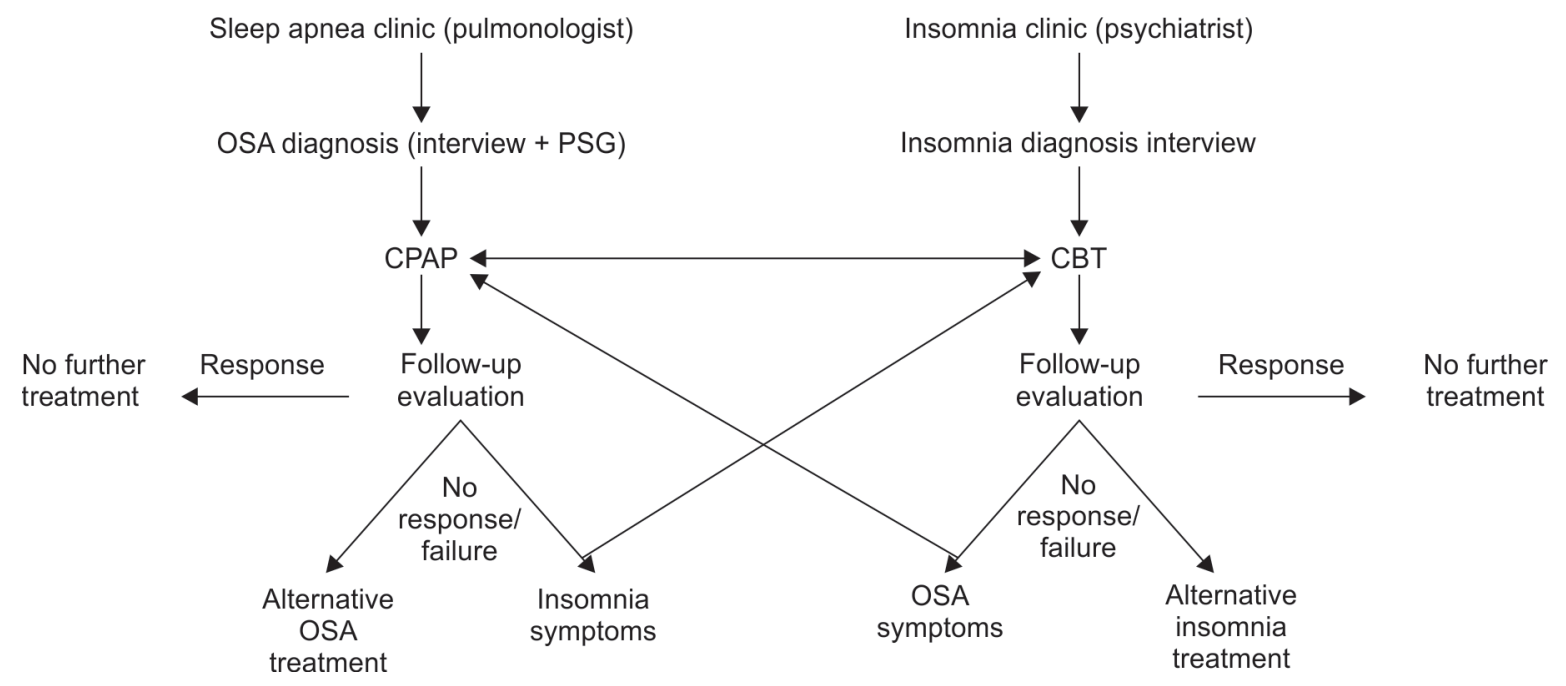

Fig. 3: Multidisciplinary approach to OSA-insomnia overlap syndrome 
$(\mathrm{SCT})^{20}$ and sleep restriction therapy (SRT). ${ }^{21} \mathrm{SCT}$ is the most widely studied and endorsed single-component treatment method. It includes instructions like (1) Go to bed when sleepy, (2) Get out of bed if not asleep in 15 minutes, (3) Repeat 1 and 2 until a rapid sleep occurs, (4) Maintain same sleep-wake time regardless of sleep length, (5) Do not nap in the day, (6) Avoid using electronic screen devices, eating in bed, worrying about losing sleep. SCT is useful for sleep onset problems. If sleep maintenance problems predominate, SRT is indicated. SRT involves restricting time in bed (minimum 5 hours) to equate with the average amount of total sleep time derived from over a week of sleep diary recordings. Sleep efficiency (percentage of time in bed asleep) is monitored weekly to attain $85 \%$ after which time in bed is gradually increased. These behavioral therapies are associated with increased risk of sleepiness in comorbid OSA patients. A novel behavior therapy, intensive sleep retraining (ISR), ${ }^{22}$ is a laboratorybased procedure which combines total sleep deprivation with many opportunities to fall asleep. As soon as sleep is attained, the patient is awoken to continue sleep deprivation and maintain high sleep drive. However, being a laboratory based test, it is labor intensive and costly. The cognitive therapies work on the principle of identifying strong held maladaptive beliefs and attempt to reduce the strength of the belief through more accurate information or experiments to test the validity of those beliefs. Both cognitive and behavior therapies are used as standalone or combined modalities for treating insomnia. ${ }^{23}$

There is no ideal approach to the OSA-insomnia overlap syndrome. Management involves addressing both the comorbidities in unison. The disease spectrum appears vast with just the tip of the iceberg being discovered so far. Current information is limited. With advancing knowledge, newer studies will pave the way for an efficient management of this new age OSA-insomnia overlap syndrome.

\section{REFERENCES}

1. Lavie P. Insomnia and sleep-disordered breathing. Sleep Medicine 2007; 8: S21-S25.

2. Ong JC, Crawford MR. Insomnia and obstructive sleep apnea. Sleep Med Clin 2013; 8: 389-398.

3. Glidewell RN. Comorbid Insomnia and sleep disordered breathing. Current treatment options in Neurology 2013; 15: 692-703.

4. Fung CH, Martin JL, Dzierzewski JM, Jouldjian D, Josephson K, Park M et al. Prevalence and Symptoms of Occult Sleep Disordered Breathing among Older Veterans with Insomnia. JCSM 2013; 9(11): 1173-1178.

5. Guilleminault C, Eldridge FL, Dement WC. Insomnia with sleep apnea: a new syndrome. Science 1973; 181: 856-858.
6. Guilleminault C, Eldridge FL, Philips JR, Dement WC. Two occult causes of insomnia and their therapeutic problems. Arch GenPsychiatr 1976; 33: 1241-1245.

7. Krakow B, Melendrez D, Ferreira E, Clark J, Warner TD, Sisley B etal. Prevalence of insomnia symptoms in patients with SDB. Chest 2001; 120: 1923-1929.

8. Sateia MJ. International Classification of Sleep Disorders -Third Edition highlights and modifications. CHEST 2014; 146 (5): 1387-1394.

9. The international classification of sleep disorders: diagnostic and coding manual. 2nd edition. American Academy of Sleep Medicine, Westchester (IL); 2005

10. Soldatos CR, Dikeos DG, Paparrigopoulos TJ. Athens Insomnia scale: validation of an instrument based on ICD-10 criteria. Journal of Psychosomatic Research 2000; 48: 555-560.

11. Bastien $\mathrm{CH}$, Vallieres A, Morin CM. Validation of the Insomnia Severity Index as an outcome measure for insomnia research. Sleep Med 2001; 2(4): 297-307.

12. Guilleminault C, Abad VC. Obstructive sleep apnea syndromes. Med Clin N Am 2004; 88: 611-630.

13. Lack LC, Sweetman AM. Diagnosis and treatment of insomnia comorbid with obstructive sleep apnea. Sleep Med Clin 2016; 11: 379-388.

14. Sweetman AM, Lack LC, Catcheside PG, Antic NA, Chai-Coetzer CL, Smith SS et al. Developing a successful treatment of co-morbid insomnia and sleep apnea. Sleep Medicine Reviews 2017; 33: 28-38.

15. Smith S, Sullivan K, Hopkins W, Douglas J. Frequency of insomnia report in patients with obstructive sleep apnoea hypopnea syndrome (OSAHS). Sleep Med 2004; 5: 449-456.

16. Krakow B, Melendrez D, Lee SA, Warner TD, Clark JO, Sklar D. Refractory Insomnia and Sleep-Disordered Breathing: A Pilot Study. Sleep and Breathing 2004; 8: 15-29.

17. Krell S, Kapur V. Insomnia complaints in patients evaluated for obstructive sleep apnea. Sleep Breath 2005; 9: 104-110.

18. Chung K. Insomnia subtypes and their relationships to daytime sleepiness in patients with obstructive sleep apnea. Respiration 2005; 72: 460-465.

19. Morin CM, Colecchi C, Stone J, Sood R, Brink D. Behavioural and pharmacological therapies for late-life insomnia: a randomized controlled trial. J Am Med Assoc 1999; 281: 991-99.

20. Bootzin RR, Epstein DR. Understanding and treating Insomnia. Annu Rev Clin Psychol 2011; 7: 435-458.

21. Spielman AJ, Saskin P, Thorpy MJ. Treatment of chronic insomnia by restriction of time in bed. Sleep 1987; 10(1): 45-56.

22. Harris J, Lack L, Kemp K, Wright H, Bootzin R. A randomised controlled trial of intensive sleep retraining (ISR): a brief conditioning treatment for chronic insomnia. Sleep 2012; 35(1): 49-60.

23. Harvey AG, Belanger L, Talbot L, Eidelman P, Bonneau SB, Brochu EF et al. Comparative efficacy of behaviour therapy, cognitive therapy and cognitive behaviour therapy for chronic insomnia: a randomised controlled trial. J Consult Clin Psychol 2014; 82(4): 670-683. 